\title{
Long gamma-ray bursts in the Swift era: the color indices of their optical afterglows
}

\section{Vojtěch Šimon*}

Astronomical Institute, The Czech Academy of Sciences, 25165 Ondřejov, Czech Republic Czech Technical University in Prague, Faculty of Electrical Engineering, Prague, Czech Republic

E-mail: simondasu.cas.cz

\section{Graziella Pizzichini}

INAF/IASF Bologna, via Gobetti 101, 40129 Bologna, Italy

E-mail: pizzichini@iasfbo.inaf.it

\section{René Hudec}

Astronomical Institute, The Czech Academy of Sciences, 25165 Ondřejov, Czech Republic Czech Technical University in Prague, Faculty of Electrical Engineering, Prague, Czech Republic

E-mail: rene.hudec@gmail.com

We present an analysis of optical afterglows (OAs) of long GRBs which were mostly discovered by NASA Swift. We used the method of the color indices that uses the commonly available multiband photometry. We show that for most OAs, in the observer frame, these colors, corrected for the reddening inside our Galaxy, display prominent clustering in some color-color diagrams. This clustering shows that we map part of the synchrotron spectrum of OAs with a very similar slope. The color indices of OAs also provide direct information about the environment of GRB. Strong concentration of most colors of OAs shows that the reddening inside their host galaxies must be quite similar and relatively small. Since OAs of the Swift GRBs are mapped in earlier phases than before, any possible color changes caused by the dust must occur in the first minutes after the start of the GRB. We show that although OAs have very similar colors indices, their absolute magnitudes display a very large scatter at the same $\left(t-T_{0}\right)_{\text {rest }}=0.1 \mathrm{~d}$ (i.e. in the rest frame of the GRB). This time corresponds to the early decay of OA. This can suggest that the properties of a fireball do not depend very much on the phenomenon which originated it while the intensities differ considerably.

XI Multifrequency Behaviour of High Energy Cosmic Sources Workshop 25-30 May 2015

Palermo, Italy

\footnotetext{
* Speaker.
} 


\section{Introduction}

Gamma-ray bursts (GRBs) are transient and unpredictable events (e.g. [7]) which are uniformly distributed in the sky [2]. A relativistic jet is the dominant source of their radiation. GRBs last from a fraction of second to several minutes. The statistical distribution of durations of GRBs shows that they can be divided into two groups - long and short ones, with the border at 2 seconds [9]. Long GRBs are interpreted as originating from hypernovae (e.g. [18]). Short GRBs may occur during a merger in a binary compact system, for example, consisting of two neutron stars [1].

Some GRBs have observable afterglows in softer bands (X-ray, optical, radio) (e.g. [7]). Also the emission of these afterglows comes from a jet. The afterglow lasts for a considerably longer time than the GRB trigger, typically for several days. The light curves of OAs usually show a dominant power-law decay, with the luminosity proportional to time as $t^{-\mathrm{a}}$.

Color indices of optical afterglows (OAs) of GRBs are a powerful approach. They help us to search for the common properties of the afterglows, specifically, to investigate the spectral energy distribution (even with relatively small telescopes with photometric filters). They also enable to search for the relations among the colors and optical luminosities of OAs. Using these indices, it is possible to constrain the properties of the interstellar medium in the host galaxy of the GRB, and also to distinguish among the individual radiation mechanisms (e.g. synchrotron radiation of the jet versus thermal emission of the supernova).

\section{Data analysis}

We used the best mapped light curve of the OA (usually in the $R$ band) for determining its profile. The color index is a difference of magnitudes measured in two bands (filters) of this curve in similar times elapsed since the GRB trigger, $t-T_{0}$.

Fig. 1 shows how the color indices of the optical afterglow can be determined. We used the best mapped light curve for determining the profile of the event. The data in the boxes were used for the determination of the color indices.

All color indices in this paper are in the observer frame. Because some OAs were observed in the $g^{\prime} r^{\prime} '{ }^{\prime}$ ' bands, we transformed them using the relation for all stars with $R-I<1.15$, published by [8]. We presented part of this analysis in [17]. Details of the determination of the color indices of OAs can be found in [14].

Because of space limitations, the reader is referred to http://gcn.gsfc.nasa.gov/ gcn/gcn3_archive.html (GCN Circulars, ed. S. Barthelmy) and J. Greiner's Web page http://www. mpe.mpg. de/ jcg/grbgen. html for bibliographic references.

Fig. 2 shows the time evolution of the color indices of OAs in the observer frame (corrected for reddening in our Galaxy using the maps of [13]). It emerges that most colors occupy a narrow belt with negligible time evolution although the brightnesses of these OAs decayed by several magnitudes. The ensemble of 25 long GRBs in the pre-Swift era is contained inside a narrow belt (a linear fit and its $\pm 1 \sigma$ are displayed) (Fig. 2a). The ensemble of long GRBs discovered by Swift consists of 32 events which constitute the belt (Fig. 2b).

Since GRBs occur in a broad range of redshifts $z$, it is desirable to examine the dependence of the color indices on $z$. Figs. 2cd show this relation for the individual ensembles. OAs with $z<3.5$ 


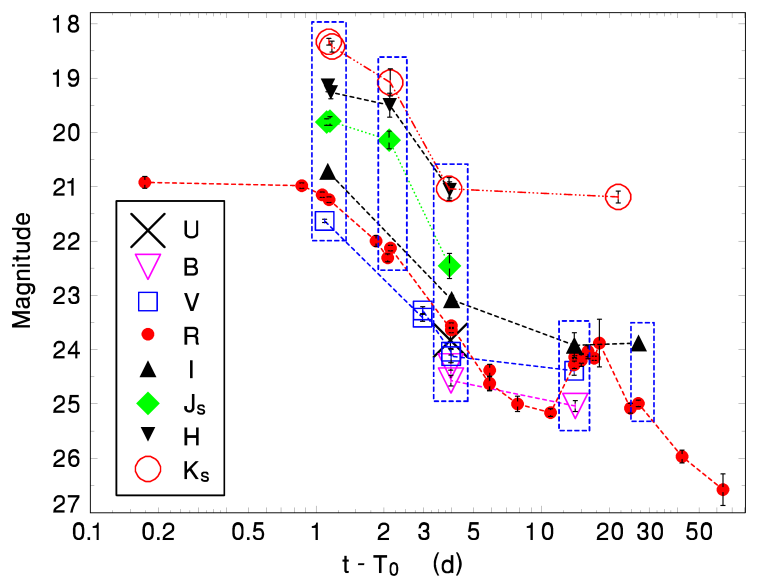

Figure 1: Example of determining the color indices of a non-uniformly mapped optical afterglow (data of [3]). The data in the boxes were used for the determination of the color indices.
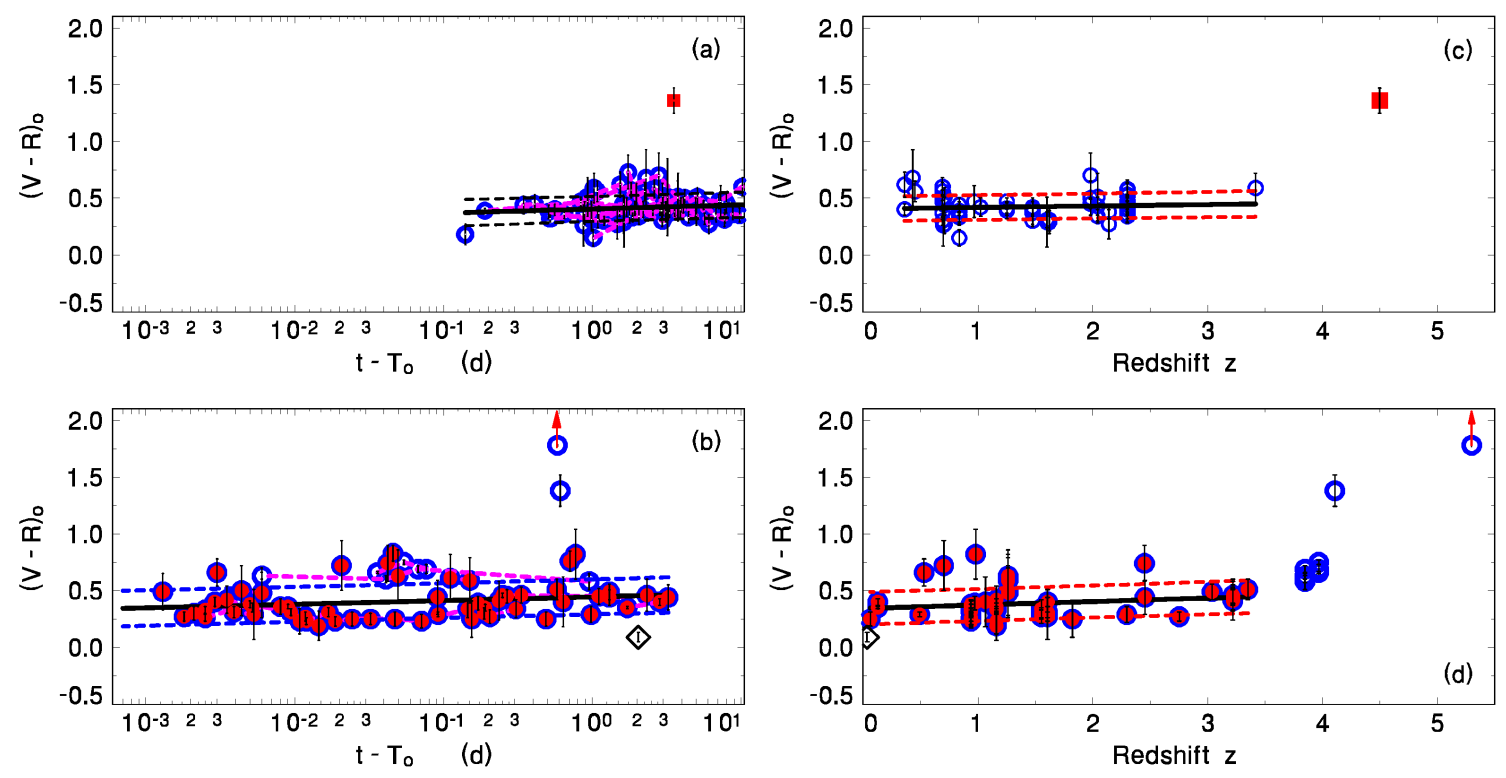

Figure 2: Time evolution of the color indices of OAs: (a) pre-Swift long GRBs (open circles with redshift $z<3.5$ are included in the fit); (b) Swift long GRBs (closed circles with redshift $z<3.5$ are included in the fit), open diamond represents OA of GRB 060218. (c-d) Relations of redshifts $z$ and the color indices: (c) pre-Swift long GRBs; (d) Swift long GRBs, open diamond represents OA of GRB 060218 and is not included in the fit.

display a very narrow range of the color, almost independent of $z$. A strong increase of the color index occurs only for high $z$, which can be explained by an increasing influence of Lyman break in the host galaxy of the GRB.

Fig. 3 shows the color-color diagram of OAs. We selected the $(V-R)_{0}$ vs. $(R-I)_{0}$ diagram because the colors of OAs displayed the strongest clustering here. The color indices are corrected for the reddening inside our Galaxy using the maps of [13]. 


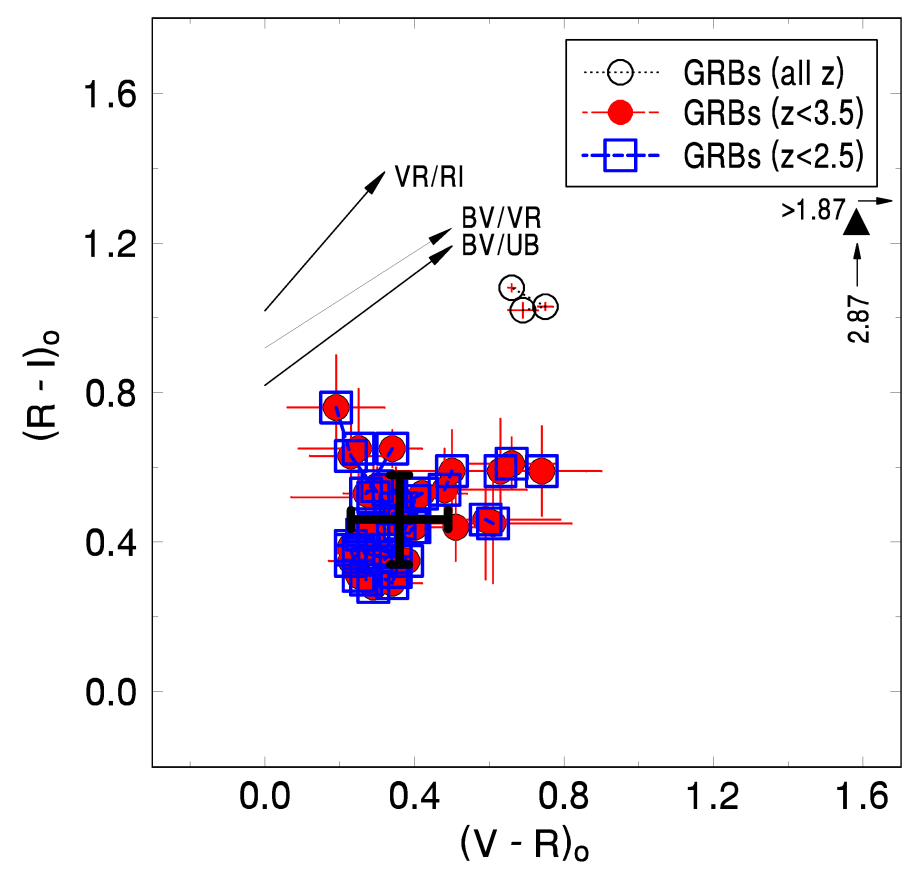

Figure 3: Color-color diagram of OAs of long GRBs discovered by Swift. The color indices are in the observer frame. The colors are corrected for the reddening inside our Galaxy using the maps of [13]. The large cross represents the mean colors (centroid) and their standard deviations of the ensemble with $z<3.5$. The arrows denote the representative reddening outside our Galaxy: $E_{\mathrm{B}-\mathrm{V}}=0.5$ mag.

The color indices of most OAs used in this paper are representative for the decaying branch of the light curve. Only in two OAs (GRB 060218 [16] and XRF 100316D [10]), the light curves displayed a strong influence (bump) of their associated supernovae on the light curves except of the early phase. Only the color indices of the early phases of these two events are therefore shown in this analysis. The mean colors (centroid) and their standard deviations of the ensemble with $z<3.5$ are displayed in Fig. 3a, too.

We also examined the range of luminosities of these OAs, for which we determined the color indices. For this purpose, we determined their absolute $R$ magnitudes using the method from our previous analysis [14]. Fig. 4 shows the statistical distributions of OAs in time $\left(t-T_{0}\right)_{\text {rest }}=0.1 \mathrm{~d}$ (i.e. in the rest frame of the GRB). The brightnesses of all of these OAs were already declining in this time. It emerges that OAs display very similar color indices although the scatter of their absolute magnitudes is very large.

\section{Results}

We present an analysis of OAs of long GRBs which were mostly discovered by NASA Swift. We used the method of the color indices that uses the commonly available multi-band photometry and which can be thus used for investigation of OAs observed by small telescopes. This approach is also applicable to very faint OAs for which spectra cannot be obtained. 


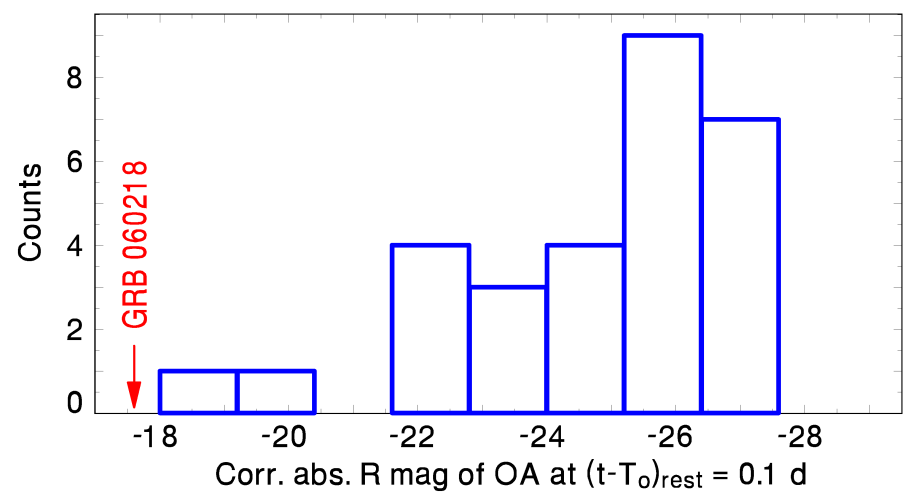

Figure 4: Absolute magnitudes of OAs of the Swift long GRBs, for which we determined the color indices. The histogram represents the early decay of brightness.

OAs of the Swift GRBs are mapped in earlier phases than was possible before. We show that for most OAs, in the observer frame, these colors, corrected for the reddening inside our Galaxy, display prominent clustering in some color-color diagrams. This clustering shows that we map part of the synchrotron spectrum of OAs with a very similar slope. The Lyman break is observed only for OAs with large $z$; for the $V-R$ index, it is only for events with $z>3.5$. Otherwise, the spectral shape of OA is very smooth, with no bumps or strong lines.

The color indices of OAs also provide direct information about the environment of GRB. Strong concentration of most colors of OAs of GRBs in Fig. 3 shows that the reddening inside their host galaxies must be quite similar and relatively small even in the early phases of the events. There are several possibilities: (a) GRBs lying on earth-watching side of a star-forming region (applicable mainly to long GRBs); (b) very low dust abundance in the host galaxy; (c) density and dust abundance of the local interstellar medium (e.g. dust from the progenitor) were reduced by intense radiation of GRB (models by [19]). We note that since OAs of the Swift GRBs are mapped in earlier phases than was possible before, the flash would have to be quite short and finish before the start of the optical observations since the observed color variations are very small.

According to [4], the dark burst fraction is constrained to be in the range 25\%-42\% for the full sample. They argue that GRBs with OAs have substantially smaller X-ray excess absorption and a substantially smaller fraction of dark bursts than the true underlying distribution. According to [5], the faint optical afterglow emission of "dark bursts" is mainly due to a combination of two contributing factors: moderate intrinsic extinction at moderate redshifts, and about $22 \%$ of "dark bursts" at $z>5$. In this regard, we note that the color-color diagram in Fig. 3 shows no tail toward redder color indices which could be ascribed to a reddening of the OA with $z<3.5$ inside its host galaxy. This suggests that we did not detect any transition between "non-reddened" OAs and "dark bursts". These two groups of OAs thus appear to be quite separated from each other and any OAs which lie between these two groups are very rare.

We show that although long GRBs produce OAs with mutually very similar colors indices, hence very similar spectral energy distributions, their absolute magnitudes display a very large scatter at the same $\left(t-T_{0}\right)_{\text {rest }}=0.1 \mathrm{~d}$ (corresponding to their early decay). This can suggest that 
the properties of a fireball do not depend very much on the phenomenon which originated it while the intensities differ considerably. Spectra of OAs in our ensembles can be interpreted by the 'fireball' model of [12]: the spectral shape in the 'fireball' model does not depend on the input energy, while the luminosity at a particular epoch does depend on it. The statistical distribution of OAs of long GRBs is skewed toward the fainter magnitudes. This may suggest the existence of faint, missed OAs.

This study was supported by grants 13-39464J and 13-33324S provided by the Grant Agency of the Czech Republic. References on OAs are given in J. Greiner's Web page www.mpe.mpg.de/ $\sim$ jcg/grbgen.html.

\section{References}

[1] D. Eichler, M. Livio, T. Piran, et al., Nature, 340, 126 (1989).

[2] G. J. Fishman, C. A. Meegan, R. B. Wilson, et al., ApJ Suppl. Series, 92, 229 (1994).

[3] J. P. U. Fynbo, J. Sollerman, J.Hjorth, et al., ApJ, 609, 962 (2004).

[4] J. P. U. Fynbo, P. Jakobsson, J. X. Prochaska, et al., ApJS, 185, 526 (2009).

[5] J. Greiner, T. Krühler, S. Klose, et al., A\&A, 526, A30 (2011).

[6] Y. F. Huang, Z. G. Dai, T. Lu, MNRAS, 332, 735 (2002).

[7] K. Hurley, R. Sari, S. G. Djorgovski, In: Compact stellar X-ray sources. Ed. by Walter Lewin \& Michiel van der Klis. Cambridge Astrophysics Series, No.39. Cambridge, UK: Cambridge University Press, p.587 (2006).

[8] S. Jester, D. P. Schneider, G. T. Richards, et al., AJ, 130, 873 (2005).

[9] C. Kouveliotou, C. A. Meegan, G. J. Fishman, et al., ApJ, 413, L101 (1993).

[10] Olivares E., F., Greiner, J., Schady, P., et al., A\&A, 539, A76 (2012).

[11] J. Rhoads, ApJ, 487, L1 (1997).

[12] R. Sari, T. Piran, R. Narayan, ApJ, 497, L17 (1998).

[13] D. J. Schlegel, D. P. Finkbeiner, M. Davis, ApJ, 500, 525 (1998).

[14] V. Šimon, R. Hudec, G. Pizzichini, N. Masetti, A\&A, 377, 450 (2001).

[15] V. Šimon, R. Hudec, G. Pizzichini, A\&A, 427, 901 (2004).

[16] V. Šimon, G. Pizzichini, R. Hudec, A\&A, 523, A56 (2010).

[17] V. Šimon, G. Pizzichini, R. Hudec, in Gamma-ray Bursts: 15 Years of GRB Afterglows. Edited by A. J. Castro-Tirado, J. Gorosabel, and I. H. Park. EAS Publications Series, Volume 61, pp.271-273 (2013).

[18] K. Z. Stanek, T. Matheson, P. M. Garnavich, et al., ApJ, 591, L17 (2003).

[19] E. Waxman, B. T. Draine, ApJ, 537, 796 (2000). 